\title{
Subjunctivity and cross-world predication
}

\author{
Kai F. Wehmeier
}

Published online: 25 January 2011

(C) The Author(s) 2011. This article is published with open access at Springerlink.com

\begin{abstract}
The main goal of this paper is to present and compare two approaches to formalizing cross-world comparisons like "John might have been taller than he is" in quantified modal logics. One is the standard method employing degrees and graded positives, according to which the example just given is to be paraphrased as something like "The height that John has is such that he might have had a height greater than it," which is amenable to familiar formalization strategies with respect to quantified modal logic. The other approach, based on subjunctive modal logic, mimics the mixed indicative-subjunctive patterns typical of cross-world comparisons in many natural languages by means of explicit mood markers. This latter approach is new and should, for various reasons, appeal to linguists and philosophers. Along the way, I argue that attempts to capture cross-world comparison by means of sentential operators are either inadequate or subject to substantive logical and philosophical objections.
\end{abstract}

Keywords Quantified modal logic - Cross-world predication · Possible worlds · Subjunctivity · Grammatical mood · Degrees · Comparatives

\section{Introduction}

The notion of cross-world comparison is perhaps best introduced by contrasting it with its intra-world cousin. Consider the sentences in groups 1 and 2 below.

(1a) John might have been taller than Mary is.

(1b) Johannes hätte größer sein können als Maria ist.

(1c) Jean aurait pu être plus grand que Marie ne l'est.

K. F. Wehmeier ( $\bowtie)$

Department of Logic and Philosophy of Science, School of Social Sciences, University of California, Irvine, 3151 Social Science Plaza, Irvine, CA 92697-5100, USA

e-mail: wehmeier@uci.edu 
(2a) John might have been taller than Mary.

(2b) Johannes hätte größer sein können als Maria.

(2c) Jean aurait pu être plus grand que Marie.

The sentences in group 1 make cross-world comparisons: They say that there is a possible world $w$ such that John, as he is in $w$, is taller than Mary, as she is in the actual world $w^{*}$. The sentences in group 2, though they are sometimes used to convey what their counterparts in group 1 express, are most naturally read as making intra-world assertions to the effect that there is a world $w$ such that in $w$, John is taller than Mary.

Standard quantified modal logic (QML) is built on the assumption that all predication is intra-world: Its predicate symbols $R$ are assigned extensions $R_{w}$ relative to each possible world $w$, and whether the relation expressed by $R$ holds of some tuple of arguments $a_{1}, \ldots, a_{n}$ at $w$ is determined by whether the argument tuple belongs to $R_{w}$. By contrast, the formalization of cross-world comparison seems to require, on the face of it, that predicate symbols be assigned extensions relative not just to one world, but across worlds.

The plan of this paper is as follows. I will first define, in Sect. 2, a notion of Kripke structure that incorporates cross-world extensions, and consider attempts to implement cross-world comparison in QML by means of sentential operators. Such attempts will turn out to be either inadequate to the task or logically and philosophically unattractive. In Sect. 3, I develop an extension of the subjunctive modal logic (SML) introduced in Wehmeier (2004) that allows for a simple and natural way of formalizing cross-world comparisons. Section 4 is dedicated to the received strategy, going back to Russell (1905), of analyzing cross-world comparison in terms of abstract objects (degrees) added to QML. This approach is based on a somewhat different notion of Kripke structure, which I define precisely. In Sect. 5, I compare the methods discussed in the preceding two sections and argue that the SML-approach developed in Sect. 3 has a leg up over the QMLbased analysis: First, it can make do without the latter's ontological commitments to abstract objects, and second, when combined with a degree-analysis, it is able to represent as substantive certain inferences that the QML-analysis must relegate to the level of informal paraphrase.

A note about terminology: By "comparison" I understand, for the purposes of this paper, the attribution of a comparative relation (strict or non-strict), or of an equivalence relation, to a pair of individuals. For the sake of exposition, I will discuss only the case of one strict cross-world comparative relation. The case of non-strict comparatives is entirely analogous, and that of equivalence relations is simpler. I will briefly indicate in the notes the modifications that would need to be made in order to cover those other cases.

\section{Cross-world extensions and sentential operators}

By a signature $\Sigma$ we will mean a pair $\Sigma=(\mathcal{C}, \mathcal{P})$, consisting of a set $\mathcal{C}$ of individual constants and a set $\mathcal{P}$ of predicate symbols. Each $P \in \mathcal{P}$ has a unique arity $\sharp P \geq 1$, 
and the binary predicate symbol $=$ is always a member of $\mathcal{P}$. A (standard) Kripke structure $\mathbb{K}$ for such a signature $\Sigma$ is a quintuple

$$
\left(K, w^{*},\left(D_{w}\right)_{w \in K},\left(c_{\mathbb{K}}\right)_{c \in \mathcal{C}},\left(P_{w}\right)_{w \in K}^{P \in \mathcal{P}}\right),
$$

where $K$ is $\mathbb{K}$ 's set of worlds; $w^{*} \in K$ is the actual world of $\mathbb{K}$; for each $w \in K, D_{w}$ is a set (the domain of individuals existing in $w$ ) such that $D:=\bigcup\left\{D_{w} \mid w \in K\right\} \neq \emptyset$; for each constant $c \in \mathcal{C}, c_{\mathbb{K}} \in D_{w^{*}}$; and for each $n$-ary predicate symbol $P \in \mathcal{P}, P_{w}$ (the extension of $P$ in $w$ ) is a subset of $D_{w}^{n}$. It is required that $=_{w}$ is true identity on $D_{w}{ }^{1}$

I will assume familiarity with the notion of a formula of the language $\mathcal{L}_{\Sigma}$ of QML for the signature $\Sigma$, as well as the notion of the truth of such a formula $\phi$ at a world $w$ of a Kripke structure $\mathbb{K}$ under some variable assignment $\sigma, \mathbb{K} \Vdash_{w} \phi[\sigma]$. Two points are perhaps worth recalling: First, by definition, $\phi$ is true in $\mathbb{K}$ (under $\sigma$ ) if it is true in $\mathbb{K}$ at the actual world $w^{*}$ of $\mathbb{K}$ (under $\sigma$ ). Second, in order to remedy certain expressive defects (of which more in Sect. 3), one often sees included in the language of QML a so-called actuality operator $A .^{2}$ This is a unary sentential operator with the property that a formula $\mathrm{A} \phi$ is true at $w$ in $\mathbb{K}$ under the assignment $\sigma$ just in case $\phi$ is true at the actual world $w^{*}$ of $\mathbb{K}$ under $\sigma$. We will assume that the language of QML contains the actuality operator, unless otherwise indicated.

The most straightforward way of interpreting cross-world predications semantically is to construe them as asserting relations between individuals in different possible worlds (typically, if not invariably, the actual world and a counterfactual one). For instance, the sentences in group (1) above make a comparison between John in some possible world $w$ and Mary in the actual world $w^{*}$. Suppose, then, that $R$ is a binary predicate intended to stand for a cross-world comparative (say the predicate of being taller than, for definiteness). Standard Kripke structures $\mathbb{K}$ assign $R$ an extension with respect to each possible world $w$, so that we may compare the individuals in any $D_{w}$ with respect to $R$. For cross-world predications, we also want to compare individuals from any $D_{v}$ with individuals in any $D_{w}$ with respect to $R$, and hence we will assign an extension $R_{v, w} \subseteq D_{v} \times D_{w}$ to $R$ for any pair $\langle v, w\rangle$ of possible worlds. To say that John, in $w$, is taller than Mary is in $w^{*}$ then just means that the pair $\left\langle j_{\mathbb{K}}, m_{\mathbb{K}}\right\rangle$ belongs to the extension $R_{w, w^{*}}$.

Formally, we proceed as follows. Let $\Sigma=(\mathcal{C}, \mathcal{P})$ be a standard signature, and let $R$ be a binary predicate symbol. A cross-world Kripke structure (CW-structure for short) $\mathbb{K}$ for $\Sigma$ with the cross-world comparative $R$ is then a sextuple

$$
\left(K, w^{*},\left(D_{w}\right)_{w \in K},\left(c_{\mathbb{K}}\right)_{c \in \mathcal{C}},\left(P_{w}\right)_{w \in K}^{P \in \mathcal{P}},\left(R_{v, w}\right)_{v, w \in K}\right),
$$

whose first five components are exactly as in the standard notion of Kripke structure. In addition, for any $v$ and $w$ in $K, R_{v, w} \subseteq D_{v} \times D_{w}$.

\footnotetext{
1 In suppressing mention of an accessibility relation, I am restricting attention to Kripke structures for the standard modal predicate logic S5. This seems reasonable, both because this semantic setting seems to be the accepted one for the metaphysical modalities under consideration in this paper, and because it illustrates the semantical and syntactical innovations against as simple a background as possible.

2 The locus classicus for the actuality operator is (Crossley and Humberstone 1977).
} 
Since $R$ is intended to be interpreted as a strict comparative across worlds, we also require that the relation $R^{*}$ defined below be a strict comparative relation on $\left\{\langle a, v\rangle \mid v \in K, a \in D_{v}\right\}$ :

$$
\langle a, v\rangle R^{*}\langle b, w\rangle: \Leftrightarrow\langle a, b\rangle \in R_{v, w} .
$$

Here, a binary relation $S$ on a set $A$ is a strict comparative if and only if it is (a) a strict partial ordering, that is, irreflexive and transitive, and (b) weakly connected on $A$ in the following sense: Whenever elements $a$ and $b$ of $A$ are not comparable with respect to $S$, then exactly the same members of $A$ bear $S$ to $a$ as do to $b$, and $a$ bears $S$ to exactly the same members of $A$ as $b$ does. ${ }^{3}$ The relevance of this constraint will become apparent later.

The first observation I wish to make is that the actuality operator is useless for the formalization of cross-world comparison, even under the restriction that one of the worlds involved must be the actual one.

This may be mildly surprising, given the tendency on the part of some writers to formulate cross-world predications with the help of the English adverb "actually," as in

John might have been taller than Mary actually is. ${ }^{4}$

A moment's reflection, however, shows that the actuality operator cannot possibly trigger our new cross-world relations. After all, the language of QML is already fully interpretable in the original kind of Kripke structure, i.e. the one without cross-world extensions. And intuitively, it is clear that there are no syntactical combinations of $\diamond, A$, and the atomic formula Rjm that would be true at a world $w$ just in case the pair $\left\langle j_{\mathbb{K}}, m_{\mathbb{K}}\right\rangle$ belongs to the cross-world extension $R_{w, w^{*}}$ : Rjm must either lie entirely within the scope of some $\mathrm{A}$, in which case the intraworld extension $R_{w^{*}, w^{*}}$ is triggered, or entirely outside the scopes of all $\mathrm{A}$, in which case the intra-world extension $R_{w, w}$, with $w$ the world of evaluation, comes into play.

Forbes $(1989$, p. 75) suggests that cross-world predications could be handled by means of a "term-binding 'actually' operator $A^{t}$," and offers the formalization $\mathrm{A}^{b} \diamond S(a, b)$ for the sentence "Your car could have been the same color mine actually is". 5 Unfortunately, Forbes does not explain the semantics of this operator; but it is clear in any case that it is unable adequately to formalize cross-world comparisons. For consider "John might have been taller than he is." Neither $\mathrm{A}^{j} \diamond R j j$ nor the perhaps slightly more plausible $\diamond \mathrm{A}^{j} R j j$ adequately formalizes it, because both occurrences of $j$ are now bound by the operator $A^{j}$.

\footnotetext{
3 If $R$ is instead intended to be interpreted as a non-strict comparative across worlds, the relation $R^{*}$ as defined above must be a non-strict comparative. Here, $S$ is a non-strict comparative on a set $A$ just in case (a) $S$ is reflexive on $A$ and transitive and (b) strongly connected on $A$, that is, whenever $a$ and $b$ are in $A$, then at least one of $a S b$ and $b S a$ holds. Finally, if $R$ is to be interpreted as an equivalence relation across worlds, the relation $R^{*}$ as defined above must be an equivalence relation.

${ }^{4}$ Cf. similar examples by Forbes (1989, p. 74), Kemp (2000, p. 305), Melia (2003, pp. 32-33), and Williamson (2006, p. 310; 2009, p. 137).

5 Essentially the same suggestion is found in (Forbes 1985, p. 93), where the term-binding actuality operator is attributed to Peacocke. In (Forbes 1994, pp. 39-40), a different operator $\mathrm{A}_{x}^{t}$, which Forbes also attributes to Peacocke, is proposed; it is essentially a variant of the operators $A_{1}$ and $A_{2}$ we consider below.
} 
Melia (2003, p. 98) notes the uselessness of the standard actuality operator for our purposes and observes:

Speaking intuitively, we want to put the A operator inside the predicate, and write something like $\diamond(R a \mathrm{~A} b)(\ldots)$.

The kind of occurrence of the actuality operator envisaged by Melia is of course not permitted by the syntax of QML. Indeed, the A-operator is a sentential operator, whereas in Melia's $\diamond(R a \mathrm{~A} b)$, it is used rather as an operator on the argument places of a predicate symbol. But the general idea of introducing an operator that acts on individual argument places seems promising and merits investigation. Its most straightforward implementation would appear to be the following.

Let $A_{1}$ and $A_{2}$ be new unary sentential operators. The semantics for these operators should imply that $\mathrm{A}_{i}$, when prefixed to an atomic formula $R a b$, forces the $i$-th argument place of $R$ to be evaluated with respect to the actual world, rather than the world of evaluation. More precisely, we want the following two clauses to hold ${ }^{6}$ :

$$
\begin{aligned}
& \left(\mathrm{A}_{1}\right) \mathbb{K} \models_{w} \mathrm{~A}_{1} R t_{1} t_{2}[\sigma] \text { iff }\left\langle\operatorname{val}_{\sigma}^{\mathbb{K}}\left(t_{1}\right), \operatorname{val}_{\sigma}^{\mathbb{K}}\left(t_{2}\right)\right\rangle \in R_{w^{*}, w} \\
& \left(\mathrm{~A}_{2}\right) \mathbb{K} \models_{w} \mathrm{~A}_{2} R t_{1} t_{2}[\sigma] \text { iff }\left\langle\operatorname{val}_{\sigma}^{\mathbb{K}}\left(t_{1}\right), \operatorname{val}_{\sigma}^{\mathbb{K}}\left(t_{2}\right)\right\rangle \in R_{w, w^{*}}
\end{aligned}
$$

In the presence of operators abiding by $\left(A_{1}\right)$ and $\left(A_{2}\right)$, we can adequately formalize our examples in group (1) as

$$
\diamond \mathrm{A}_{2} \operatorname{Rjm},
$$

as is easily verified.

Reasonable as the introduction of $A_{1}$ and $A_{2}$ may seem, and notwithstanding their success in assigning formalizations with the right truth conditions to the sentences in group (1), there are reasons to be dissatisfied with these operators.

First, they are hyperintensional ${ }^{7}$ : necessary equivalence of formulas is no guarantee that they will be intersubstitutible in $\mathrm{A}_{i}$-contexts. For instance, if $R$ and $S$ are both strict cross-world comparatives (say $R$ stands for "richer than" and $S$ for "smarter than"), the sentence $\square(R a a \leftrightarrow S a a)$ is true in every model, while models exist in which $\diamond \mathrm{A}_{2} R a a$ is true and $\diamond \mathrm{A}_{2} S a a$ false. That cross-world comparison should force us to introduce hyperintensional operators into an antecedently nonhyperintensional language speaks against this approach.

Hyperintensionality, however, is not the only problem with the $A_{i}$. So far, we have specified the truth conditions for formulas $A_{i} \phi$ only for the case where $\phi$ is of the form $R t_{1} t_{2}$; but in order for $\mathrm{A}_{i}$ to be a proper logical operator, we must state the semantic clause for formulas of the form $\mathrm{A}_{i} \phi$ generally. ${ }^{8}$ The only case where we have strong intuitions as to the intended behavior of the operators $A_{i}$ at all is when

\footnotetext{
${ }^{6}$ Here val ${ }_{\sigma}^{\mathbb{K}}(t)$ is the value of the term $t$ in the structure $\mathbb{K}$ under the variable assignment $\sigma$. Cf. Definition 2 below.

7 I owe this observation to Tim Williamson.

8 Indeed, if at all possible, the semantic clause for $\mathrm{A}_{i} \phi$ should be stated independently of the form of the argument $\phi$. But this desideratum simply cannot be met. Consider, for instance, the relatively simple cases of $\mathrm{A}_{1}(\phi \wedge \psi)$ and $\mathrm{A}_{1} R j m$. We know that $\mathrm{A}_{1} R j m$ is to be true at $w$ just in case $\left(j_{\mathbb{K}}, m_{\mathbb{K}}\right) \in R_{w^{*}, w}$, but there is nothing analogous we could require for the truth of $\mathrm{A}_{1}(\phi \wedge \psi)$ at $w$, nor does there seem to be a general clause that could subsume that for $A_{1}$ Rjm and whichever one we pick for $A_{1}(\phi \wedge \psi)$.
} 
they occur in front of an atomic formula $R t_{1} t_{2}$; but under which conditions $\mathrm{A}_{i}(\phi \wedge \psi)$, say, should be true at a world $w$ is entirely up for grabs. We could stipulate this to be equivalent to the truth of both $\mathrm{A}_{i} \phi$ and $\mathrm{A}_{i} \psi$ at $w$, or to be always false (or true), or equivalent to $\phi \wedge \psi$. The difficulties of even settling on a general semantics for $A_{1}$ and $A_{2}$ suggest that these devices aren't really sentential operators at all. ${ }^{9}$ We will see in a moment that the $A_{i}$ are really predicate markers (for indicative mood) in disguise.

\section{Cross-world subjunctive modal logic}

In Wehmeier (2004), I considered sentences like the following:

(3a) Under certain circumstances, everybody who is rich would have been poor.

(3b) Es hätte sein können, daß jeder, der reich ist, arm gewesen wäre.

(3c) Il aurait pu se faire que tous ceux qui sont riches soient pauvres.

The natural reading for $(3 a)-(3 c)$, in possible-worlds jargon, seems to be that there exists a world $w$ such that everyone who is rich in the actual world, $w^{*}$, is poor in $w$. As it turns out, there is no sentence in the language of QML (without the actuality operator) that adequately formalizes this reading (cf. Wehmeier 2003).

Indeed, a moment's reflection leads one to suspect as much. One would expect a formalization of (3a)-(3c) to result from the skeleton $\forall x(R x \rightarrow P x)$ by the insertion of a diamond. Now

$$
\diamond \forall x(R x \rightarrow P x)
$$

is inadequate, because once the diamond has sent us to some possible world $w$, the sentence $\forall x(R x \rightarrow P x)$ is evaluated there; however, $R x$ must be evaluated at $w^{*}$ to capture the reading at hand. The sentence

$$
\forall x \gg(R x \rightarrow P x)
$$

fails for the same reason, but has the additional drawback that it doesn't require one possible world that works for all individuals (the world required to exist by the diamond might depend on $x$ ). In other words, we have here a $\forall \exists$-combination, while the reading we're interested in requires an $\exists \forall$-combination. This is also why

$$
\forall x(R x \rightarrow \diamond P x)
$$

fails as a formalization of (3a)-(3c), even though, in this case, the predicate $R x$ does contribute $R_{w^{*}}$, its extension at $w^{*}$, to the sentence's truth condition.

This expressive deficit is clearly due to the fact that in the language of QML, absent the actuality operator, there is only one way of ensuring that a predicate be evaluated at $w^{*}$ rather than at the world of evaluation: by having the predicate occur outside of the scopes of all modal operators. This suggests that the indicativesubjunctive distinction is not exhaustively explained by correlating indicative

\footnotetext{
9 One could also try to define operators $S_{1}$ and $S_{2}$ that stand to Humberstone's (1982) operator $S$ (cf. Sect. 3) as $A_{1}$ and $A_{2}$ stand to $A$. But analogous considerations speak against the status of the $S_{i}$ as fullfledged operators.
} 
predicates with those lying outside the scopes of modal operators, and subjunctive ones with those that occur within such scopes. ${ }^{10}$ In sentences (3a)-(3c), for instance, one wants the indicative predicates ("is rich," "reich ist," "sont riches") to lie within the scope of the governing possibility operator.

Humberstone (1982) observed that this expressive deficit could be overcome by introducing a subjunctivity operator $\mathrm{S}$. This approach is very similar in spirit to the method of subjunctive markers I developed independently much later (Wehmeier $2004,2005)$ in that both $S$ and my subjunctive marker signal the presence of a bindable world-variable. My main objection to Humberstone's formalism is that $S$ is syntactically a sentential operator, but cannot be allowed the freedom of syntactic occurrence characteristic of such operators. It would seem that this objection is confirmed in the present context by the considerations adduced in footnote 9 above. ${ }^{11}$

My own proposal, in any case, was to model the indicative-subjunctive distinction in the object language by making a predicate's mood syntactically explicit, using mood markers " $i$ " (for "indicative") and " $s$ " (for "subjunctive"). ${ }^{12}$ This leads to the following:

Definition 1 Let $\Sigma=(\mathcal{C}, \mathcal{P})$ be a signature. The terms of the associated language $\mathcal{L}_{\Sigma}^{s}$ of subjunctive modal logic (SML) are the individual variables $\left(x, y, z, x_{0}, x_{1}, \ldots\right)$, and the individual constants from $\mathcal{C}$. If $R$ is an $n$-ary predicate symbol from $\mathcal{P}$, and $t_{1}, \ldots, t_{n}$ are terms of $\mathcal{L}_{\Sigma}^{s}$, then both $R^{i} t_{1} \ldots t_{n}$ and $R^{s} t_{1} \ldots t_{n}$ are atomic formulas of $\mathcal{L}_{\Sigma}^{s}$. We sometimes refer to $R^{i}$ as an indicative predicate, and to $R^{s}$ as a subjunctive predicate. The formulas of $\mathcal{L}_{\Sigma}^{s}$ are generated from its atomic formulas by means of the propositional connectives, the modal operators $\square$ and $\diamond$, the indicative quantifiers $\forall^{i}$ and $\exists^{i}$, as well as the subjunctive quantifiers $\forall^{s}$ and $\exists^{s}$. A formula is individually closed if it contains no free occurrences of individual variables, and subjunctively closed if every subjunctive marker " $s$ " in the formula occurs within the scope of some modal operator. A formula is a sentence if it is both individually and subjunctively closed.

Before we move on to the semantics for $\mathcal{L}_{\Sigma}^{s}$, some explanations may be in order.

(a) The mood distinction for quantifiers, first introduced in (Wehmeier 2005), is needed in order to deal with varying domains in Kripke structures (much like the actuality quantifiers of Hazen 1990). The idea that quantifiers can occur in both indicative and subjunctive mood is not absurd: In English, we certainly have both indicative ("there exist," "there are") and subjunctive ("there would have existed," "there would have been") existential quantifiers.

\footnotetext{
${ }^{10}$ It might have been more appropriate to speak of the realis-irrealis distinction rather than the indicative-subjunctive distinction, as a number of moods can serve to express the irrealis, such as the conditional and subjunctive moods in English, the conditionnel and subjonctif in French, and the Konjunktiv II in German. I stick with the indicative-subjunctive terminology mostly because it is somewhat entrenched in the logical literature.

11 See also (Wehmeier 2005, p. 200), as well as the discussion in (Humberstone 2004, pp. 46-49).

12 In earlier work, I used only the subjunctive marker " $s$ " and regarded unmarked predicates as indicative. This has certain notational disadvantages for the case of cross-world predication. Clearly the switch to marking both moods is immaterial, as the resulting languages are trivial variants of each other.
} 
(b) In ordinary predicate logic, individually closed formulas are of special interest because their truth values do not depend on the choice of a variable assignment; they are true or false simpliciter. Subjunctively closed formulas are, as we will see shortly, true or false regardless of the choice of a world of evaluation, and thus sentences in the sense of Definition 1 are true or false simpliciter (relative neither to a choice of values for the variables nor to the choice of a world of evaluation).

(c) QML with actuality operator (and actuality quantifiers) is expressively equivalent to SML in the sense that for every sentence of one system, there is a sentence of the other that has the same class of Kripke models. Nevertheless, the two systems are not simply notational variants of each other. See (Wehmeier 2005).

Next, the semantics for $\mathcal{L}_{\Sigma}^{s}$.

Definition 2 Let $\mathbb{K}=\left(K, w^{*},\left(D_{w}\right)_{w \in K},\left(c_{\mathbb{K}}\right)_{c \in \mathcal{C}},\left(P_{w}\right)_{w \in K}^{P \in \mathcal{P}}\right)$ be a standard Kripke structure for $\Sigma=(\mathcal{C}, \mathcal{P})$. Let $\sigma$ be a variable assignment, that is, a function from the individual variables into $D=\bigcup_{w \in K} D_{w}$. For any variable $x$ and $a \in D, \sigma(x / a)$ is the $x$-variant of $\sigma$ that maps $x$ to $a$. Where $x$ is an individual variable, we let val $\left.\right|_{\sigma} ^{\mathbb{K}}(x)$ be $\sigma(x)$, and where $c \in \mathcal{C}$, we put val $\left.\right|_{\sigma} ^{\mathbb{K}}(c):=c_{\mathbb{K}}$. In this way, we have specified a value val $_{\sigma}^{\mathbb{K}}(t) \in D$ for every term $t$ of $\mathcal{L}_{\Sigma}^{s}$. Next, we define the notion of truth in a Kripke structure with respect to a variable assignment $\sigma$ and a salient possible world $w$ by recursion as follows:

- $\quad \mathbb{K} \models_{w} R^{i} t_{1} \ldots t_{n}[\sigma]$ iff $\left\langle\mathrm{val}_{\sigma}^{\mathbb{K}}\left(t_{1}\right), \ldots, \mathrm{val}_{\sigma}^{\mathbb{K}}\left(t_{n}\right)\right\rangle \in R_{w^{*}}$ (that is, indicative predicates are evaluated at the actual world, no matter what the salient possible world is);

- $\quad \mathbb{K} \models_{w} R^{s} t_{1} \ldots t_{n}[\sigma]$ iff $\left\langle\mathrm{val}_{\sigma}^{\mathbb{K}}\left(t_{1}\right), \ldots, \mathrm{val}_{\sigma}^{\mathbb{K}}\left(t_{n}\right)\right\rangle \in R_{w}$ (that is, subjunctive predicates are always evaluated at the salient possible world);

- $\mathbb{K} \models_{w} \phi \wedge \psi[\sigma]$ iff $\mathbb{K} \models_{w} \phi[\sigma]$ and $\mathbb{K} \models_{w} \psi[\sigma]$, and similarly for the other propositional connectives;

- $\quad \mathbb{K} \models_{w} \forall^{i} x \phi[\sigma]$ iff for every $a \in D_{w^{*}}, \mathbb{K} \models_{w} \phi[\sigma(x / a)]$, and similarly for $\exists^{i}$ (that is, the indicative quantifiers range over the domain of the actual world, no matter what the salient possible world is);

- $\mathbb{K} \models_{w} \forall^{s} x \phi[\sigma]$ iff for every $a \in D_{w}, \mathbb{K} \models_{w} \phi[\sigma(x / a)]$, and similarly for $\exists^{s}$ (that is, the subjunctive quantifiers range over the domain of the salient possible world);

- $\quad \mathbb{K} \models_{w} \diamond \phi[\sigma]$ iff for some $v \in K, \mathbb{K} \models_{v} \phi[\sigma]$;

- $\mathbb{K} \models_{w} \square \phi[\sigma]$ iff for all $v \in K, \mathbb{K} \models_{v} \phi[\sigma]$.

It is easy to see that for individually closed $\phi$, whether or not $\mathbb{K} \models_{w} \phi[\sigma]$ is independent of $\sigma$, so for such formulas we may define $\mathbb{K} \models_{w} \phi$ as, say, $\mathbb{K} \models_{w} \phi[\sigma]$ for every variable assignment $\sigma$. Similarly, for subjunctively closed formulas $\phi$, whether or not $\mathbb{K} \models_{w} \phi[\sigma]$ is independent of $w$, and so for such formulas we may define $\mathbb{K} \models \phi[\sigma]$ as, say, $\mathbb{K} \models_{w} \phi[\sigma]$ for all $w \in K$. For sentences $\phi$ we thus have a notion of truth in $\mathbb{K}$ simpliciter, namely $\mathbb{K} \models \phi$ iff for every variable assignment $\sigma$ and every world $w \in K, \mathbb{K} \models_{w} \phi[\sigma]$. 
The logic determined by these syntactic and semantic definitions will be called subjunctive modal logic or SML, following the terminology of (Wehmeier 2004). It is easy to see that the SML-sentence $\diamond \forall^{i} x\left(R^{i} x \rightarrow P^{s} x\right)$ formalizes the sentences in group (3) above. We have:

$$
\mathbb{K} \models \diamond \forall^{i} x\left(R^{i} x \rightarrow P^{s} x\right)
$$

iff for every $\sigma$ and $w, \mathbb{K} \models_{w} \diamond \forall^{i} x\left(R^{i} x \rightarrow P^{s} x\right)[\sigma]$

iff for every $\sigma$ and $w$, there is a $v$ such that $\mathbb{K} \models_{v} \forall^{i} x\left(R^{i} x \rightarrow P^{s} x\right)[\sigma]$

iff for every $\sigma$ there is a $v$ such that for all $a \in D_{w^{*}}, \mathbb{K} \models_{v} R^{i} x \rightarrow P^{s} x[\sigma(x / a)]$

iff for every $\sigma$ there is a $v$ such that for all $a \in D_{w^{*}}$, if $\mathbb{K} \models_{v} R^{i} x[\sigma(x / a)]$, then $\mathbb{K} \models_{v} P^{s} x[\sigma(x / a)]$

iff for every $\sigma$ there is a $v$ such that for all $a \in D_{w^{*}}$, if $\sigma(x / a)(x) \in R_{w^{*}}$, then $\sigma(x / a)(x) \in P_{v}$

iff there is a $v$ such that for all $a \in D_{w^{*}}$, if $a \in R_{w^{*}}$, then $a \in P_{v}$

iff there is a $v$ such that $R_{w^{*}} \subseteq P_{v}$,

which is exactly the desired truth condition.

In order to see how cross-world predication can be implemented in the syntax of SML, let us consider the following examples:

(4a) Under certain circumstances, John would have been taller than Mary is.

(4b) Es hätte sein können, dass Johannes größer wäre als Maria ist.

(4c) Il aurait pu se faire que Jean soit plus grand que Marie ne l'est.

We see that the cross-world predicates all have two copulas that can be individually inflected for mood: "would have been" is conditional mood, "wäre" is Konjunktiv II, and "soit" is subjonctif; by contrast, "is," "ist," and "est" are all indicative. This suggests equipping a predicate symbol intended for cross-world comparison with two mood markers rather than just one. In other words, if we add a binary predicate symbol $R$ to the language of SML with the intention of using it for cross-world comparison, the definition of the formulas is to be supplemented by the following stipulation:

If $t_{1}, t_{2}$ are terms, then the following are all formulas:

1. $R^{i, i} t_{1} t_{2}$

2. $R^{i, s} t_{1} t_{2}$

3. $R^{s, i} t_{1} t_{2}$

4. $R^{s, s} t_{1} t_{2}$

Now we just need to connect this syntactic innovation up with the cross-world extensions included in the new type of Kripke structure; but this is easy. We define:

- $\quad \mathbb{K} \models_{w} R^{i, i} t_{1} t_{2}[\sigma]$ iff $\left\langle\operatorname{val}_{\sigma}^{\mathbb{K}}\left(t_{1}\right)\right.$, val $\left._{\sigma}^{\mathbb{K}}\left(t_{2}\right)\right\rangle \in R_{w^{*}, w^{*}}$

- $\quad \mathbb{K} \models_{w} R^{i, s} t_{1} t_{2}[\sigma]$ iff $\left\langle\operatorname{val}_{\sigma}^{\mathbb{K}}\left(t_{1}\right)\right.$, val $\left._{\sigma}^{\mathbb{K}}\left(t_{2}\right)\right\rangle \in R_{w^{*}, w}$

- $\quad \mathbb{K} \models_{w} R^{s, i} t_{1} t_{2}[\sigma]$ iff $\left\langle\operatorname{val}_{\sigma}^{\mathbb{K}}\left(t_{1}\right)\right.$, val $\left._{\sigma}^{\mathbb{K}}\left(t_{2}\right)\right\rangle \in R_{w, w^{*}}$

- $\quad \mathbb{K} \models_{w} R^{s, s} t_{1} t_{2}[\sigma]$ iff $\left\langle\operatorname{val}_{\sigma}^{\mathbb{K}}\left(t_{1}\right), \operatorname{val}_{\sigma}^{\mathbb{K}}\left(t_{2}\right)\right\rangle \in R_{w, w}$ 
The logical system thus obtained will be called CSML ("cross-world SML"). The reader may wish to check for herself that we can adequately formalize the sentences in groups (1) and (4) in CSML as

$$
\diamond R^{s, i} j m \text {. }
$$

It should be noted that the use of the two mood markers $i$ and $s$ does not exploit the full resources for cross-world predication that the $\mathrm{CW}$-structures provide, because we are currently modeling only cross-world predications one of whose worlds is $w^{*}$. The majority of cross-world predications that occur in English, German, or French seem to be of this type. There are, however, natural-language statements that require additional logical resources. Consider

(5) Under certain circumstances, everybody would have been as generous as they might have been.

I take it that (5) can be analyzed, in possible-worlds parlance, as saying that there exists a world $w$ such that for every world $v$, it is not the case that some individual from $w$ is more generous in $v$ than she is in $w$; in other words, $w$ is a world of maximal generosity. CSML, as we have developed it so far, cannot directly accommodate (5), because the only cross-world comparisons it is equipped to handle are those that involve the actual world. But (5) requires a cross-world comparison between two merely possible worlds.

It is not difficult, however, to see how CSML can be extended to a system that provides logical forms for sentences such as (5): Add to the language of CSML indexed modal operators $\square_{1}, \square_{2}, \diamond_{1}, \diamond_{2}$ as well as indexed quantifiers $\forall_{1}, \forall_{2}, \exists_{1}, \exists_{2}$, and allow additional atomic formulas $R^{s_{j}, s_{k}} t_{1} t_{2}, R^{s_{j}, i} t_{1} t_{2}$, and $R^{i, s_{j}} t_{1} t_{2}$ (for $j, k \in\{1,2\}$ ). Evaluation of a formula will now be relative to two worlds of evaluation (corresponding to the two subjunctive markers $s_{1}$ and $s_{2}$ ), as follows: $\mathbb{K} \models_{w}^{v} R^{s_{1}, s_{2}} t_{1} t_{2}[\sigma]$ iff $\left\langle\operatorname{val}_{\sigma}^{\mathbb{K}}\left(t_{1}\right)\right.$, val $\left._{\sigma}^{\mathbb{K}}\left(t_{2}\right)\right\rangle \in R_{v, w}$, and analogously for the other new atomic formulas. The indexed modal operators quantify into the upper and lower world index, respectively, of the double turnstile, e.g. thus: $\mathbb{K} \models_{w}^{v} \diamond_{2} \phi[\sigma]$ iff for some world $u \in K, \mathbb{K} \models_{u}^{v} \phi[\sigma]$. The quantifier $\forall_{1}$ will range over the individuals existing at the upper world index, $\forall_{2}$ over those at the lower world index, and similarly for the existential versions. We can then formalize (5) in this extended language as

$$
\diamond_{1} \forall_{1} x \neg \diamond_{2} R^{s_{2}, s_{1}} x x \text {. }
$$

In an analogous fashion, one can allow for any finite number or even infinitely many indexed subjunctive markers, modal operators, and quantifiers.

\section{Cross-world comparison via degrees}

As far as I am aware, the only method of dealing with cross-world predication that has been discussed in any detail in the literature is the approach, going back to Russell (1905), that employs quantification over certain abstract objects (degrees) 
associated with the predicates attributed across worlds. Its origin is Russell's anecdote of the irritable yachtsman:

I have heard of a touchy owner of a yacht to whom a guest, on first seeing it, remarked, "I thought your yacht was larger than it is"; and the owner replied, "No, my yacht is not larger than it is". What the guest meant was, "The size that I thought your yacht was is greater than the size your yacht is"; the meaning attributed to him is, "I thought the size of your yacht was greater than the size of your yacht”. (Russell 1905, p. 489)

As Kripke (2005, p. 1022) has pointed out, Russell botches his own joke, in that he attributes an overly specific belief to the guest. Briefly: Where $L x$ is the predicate, "the yacht has size $x$," and B is the operator "the guest believes that," Russell explains the misunderstanding by disambiguating the guest's statement into

(a) [the $x: \mathrm{B} L x][$ the $y: L y] x>y$, which, according to Russell, is what the guest meant, and

(b) $\mathrm{B}([$ the $x: L x][$ the $y: L y] x>y)$, "the meaning attributed to him" by the yachtsman.

Kripke notes that (a) fails adequately to formalize the guest's statement, because it implies that there is a unique size of which the guest believed that the yacht had it, which is clearly not required for the truth of the guest's remark. It is perhaps also worth pointing out that the difference between (a) and (b) is in no way due to a scope ambiguity of definite descriptions-(a) is not a wide-scope version of (b); in fact, the descriptive scopes are maximally narrow in both (a) and (b). What makes (a) differ from (b) is rather that the former contains substantively distinct descriptions ("the $x$ : B $L x$ " and "the $y$ : $L y$ "), whereas the descriptions occurring in the latter are merely alphabetic variants of each other.

Be that as it may, one can fruitfully bring to bear Russellian scope considerations on the issue, by introducing a mixed-scope reading of (b) above: The formulas

$\left(\mathrm{a}^{\prime}\right) \quad([$ the $y: L y] \mathrm{B}[$ the $x: L x] x>y)$ and

$\left(\mathrm{b}^{\prime}\right) \quad \mathrm{B}([$ the $x: L x][$ the $y: L y] x>y)$

correctly formalize the two readings of the guest's ambiguous statement. ${ }^{13}$

It should be clear how this method can be adapted to enable formalization of the sentences in groups (1) and (4) in QML. We first paraphrase them as

(6) Mary's height is such that it might have been the case that John's height would have been greater than it,

and then directly formalize (6) in QML as follows:

$\left(6^{\prime}\right)[$ the $x: T m x] \diamond[$ the $y: T j y] y>x$,

where Tax means that individual $a$ has height $x$, and the bound variables range over heights.

13 Kripke (2005, p. 1023) attributes this reconstruction to Nathan Salmon. 
In general, the Russellian approach will require an appeal to the degrees to which an individual might possess the positive that corresponds to the comparative attributed across worlds; in the case of our example, we need to invoke heights as the degrees to which an individual might be tall. Concomitantly, the modal language will need to be extended to include a predicate for the graded positive (in the example, Tax for " $a$ is $x$ tall").

To put this method on a firmer footing, let us consider how to accommodate such degrees and graded positives in a Kripke structure. The degrees belonging to a comparative (whether strict or non-strict) should obviously be linearly ordered. Further, every degree of $R$-ness is presumably a degree that some individual might have had, which is to say that for every degree $d$, there should be a world $w$ in which some individual $a$ possesses $R$-ness to degree $d$.

These informal considerations suggest the following formal definition:

Definition 3 A degree-structure (D-structure for short) $\mathbb{K}$ for a signature $\Sigma=(\mathcal{C}, \mathcal{P})$ and one strict cross-world comparative $R$ is a septuple

$$
\left(K, w^{*},\left(D_{w}\right)_{w \in K},\left(c_{\mathbb{K}}\right)_{c \in \mathcal{C}},\left(P_{w}\right)_{w \in K}^{P \in \mathcal{P}},\left(D_{R},>_{R}, f_{R}\right),\left(T_{w}\right)_{w \in K}\right),
$$

whose first five components are exactly as in the standard notion of Kripke structure. In addition, $D_{R}$ (the set of $R$-degrees) is a non-empty set strictly linearly ordered by $>_{R}$, and $f_{R}$ is a function onto $D_{R}$ that maps each pair $\langle a, w\rangle$ with $a \in D_{w}$ to some $f_{R}(a, w) \in D_{R}$. Further, for each world $w \in K, T_{w}$ is a subset of $D_{w} \times D_{R}$ (the extension, at $w$, of the graded positive belonging to $R$ ), in such a way that $\langle a, d\rangle \in$ $T_{w}$ if and only if $f_{R}(a, w)=d .^{14}$

The most convenient way of implementing Russellian cross-world comparison in the language of QML would then seem to be this: Add a new sort of individual variables (say $h_{0}, h_{1}, \ldots$ ) as primitive symbols whose values are restricted to the members of $D_{R}$, and add a new binary predicate symbol $T$ whose first argument place may only be filled with terms of the original sort, and whose second argument place only accommodates terms of $R$-degree sort. Bound variables of degree sort will vary over the entire set $D_{R}$ independently of the world of evaluation; i.e. $\mathbb{K} \models_{w} \forall h \phi[\sigma]$ will hold if and only if for all $d \in D_{R}, \mathbb{K} \models_{w} \phi[\sigma(h / d)]$. The sentences in groups (1) and (4) can then be literally formalized in QML by the formula $\left(6^{\prime}\right)$, which receives the desired truth conditions under the semantics just described.

Given the intertranslatability of QML and SML mentioned above, it is clear that a Russellian analysis could just as well be carried out in the context of SML. What is perhaps remarkable, however, is that the Russellian degree analysis appears to be the only way of allowing for cross-world comparisons in QML, whereas SML can be extended both by Russellian degrees and into the system CSML. I will comment on this situation in more detail below.

\footnotetext{
14 In the case of a non-strict cross-world comparative, one would require $>_{R}$ to be a non-strict linear ordering on $D_{R}$. In the equivalence relation case, no ordering of the degrees would be required.
} 


\section{Comparison of approaches}

In trying to assess the relative merits of the different ways of implementing crossworld predication, it is important to distinguish two dimensions of comparison: First, we may ask whether CW-structures or D-structures are the more appropriate notion of Kripke structure when it comes to cross-world predication; second, we may ask whether an approach based on QML is preferable to one based on SML, or vice versa.

As regards the first question, there is a sense in which the notions of model are equivalent (modulo a first-order abstraction principle): Each D-structure uniquely determines a $\mathrm{CW}$-structure and conversely. This can be seen as follows.

First, let $\mathbb{K}$ be the D-structure

$$
\left(K, w^{*},\left(D_{w}\right)_{w \in K},\left(c_{\mathbb{K}}\right)_{c \in \mathcal{C}},\left(P_{w}\right)_{w \in K}^{P \in \mathcal{P}},\left(D_{R},>_{R}, f_{R}\right),\left(T_{w}\right)_{w \in K}\right) .
$$

For each pair $\langle v, w\rangle$ of worlds from $K$, define $R_{v, w}$ to be the set of all pairs $\langle a, b\rangle$ with $f_{R}(a, v)>_{R} f_{R}(b, w)$. Then $\mathbb{K}^{\prime}$ defined as follows:

$$
\left(K, w^{*},\left(D_{w}\right)_{w \in K},\left(c_{\mathbb{K}}\right)_{c \in \mathcal{C}},\left(P_{w}\right)_{w \in K}^{P \in \mathcal{P}},\left(R_{v, w}\right)_{v, w \in K}\right)
$$

is a CW-structure for the same signature as $\mathbb{K}$. To verify this, we need only show that the relation $R^{*}$ as defined below is a strict comparative on the set $\left\{\langle a, v\rangle \mid v \in K, a \in D_{v}\right\}$ :

$$
\langle a, v\rangle R^{*}\langle b, w\rangle: \Leftrightarrow\langle a, b\rangle \in R_{v, w} .
$$

By definition, $\langle a, b\rangle \in R_{v, w}$ iff $f_{R}(a, v)>_{R} f_{R}(b, w)$. Hence irreflexivity and transitivity of $R^{*}$ follow from irreflexivity and transitivity of $>_{R}$. It remains to establish the weak connectedness of $R^{*}$. So suppose that neither $\langle a, b\rangle \in R_{v, w}$ nor $\langle b, a\rangle \in R_{w, v}$. Then neither $f_{R}(a, v)>_{R} f_{R}(b, w)$ nor $f_{R}(b, w)>_{R} f_{R}(a, v)$ and hence, by the connectedness of $>_{R}, f_{R}(a, v)=f_{R}(b, w)$. It follows that $\langle a, v\rangle$ bears $R^{*}$ to the same pairs as $\langle b, w\rangle$ does and that the same pairs bear $R^{*}$ to $\langle a, v\rangle$ as do to $\langle b, w\rangle$, and hence $R^{*}$ is weakly connected in the requisite sense. ${ }^{15}$

Now let $\mathbb{K}$ be the $\mathrm{CW}$-structure

$$
\left(K, w^{*},\left(D_{w}\right)_{w \in K},\left(c_{\mathbb{K}}\right)_{c \in \mathcal{C}},\left(P_{w}\right)_{w \in K}^{P \in \mathcal{P}},\left(R_{v, w}\right)_{v, w \in K}\right) .
$$

In particular, then, the relation $R^{*}$ defined as above must be a strict comparative on $\left\{\langle a, v\rangle \mid v \in K, a \in D_{v}\right\}$. From $R^{*}$, we can define an equivalence relation $\sim_{R}$ as follows:

$$
\langle a, v\rangle \sim_{R}\langle b, w\rangle: \Leftrightarrow \neg\langle a, v\rangle R^{*}\langle b, w\rangle \wedge \neg\langle b, w\rangle R^{*}\langle a, v\rangle .
$$

Since elements incomparable with respect to a strict comparative on a given domain must compare in the same way to all elements of the domain, this is tantamount to saying that the pairs $\langle a, v\rangle$ and $\langle b, w\rangle$ are $\sim_{R}$-equivalent just in case

\footnotetext{
$\overline{15}$ Similar considerations apply in the case of non-strict comparatives. For cross-world equivalence relations $R$, one would define $R_{v, w}$ as the set of all pairs $\langle a, b\rangle$ with $f_{R}(a, v)=f_{R}(b, w)$. It is then obvious that $R^{*}$ must be an equivalence relation.
} 
they are indiscernible with respect to $R^{*}$. Reflexivity, symmetry, and transitivity of $\sim_{R}$ follow readily.

Now we will appeal to the following abstraction principle:

(Abst) With every equivalence relation $\approx$ on a set $D$ is associated a function $f \approx$ with domain $D$ such that

$$
\forall x \forall y\left(x \approx y \leftrightarrow f_{\approx}(x)=f_{\approx}(y)\right) .
$$

Taking $\left\{\langle a, v\rangle \mid v \in K, a \in D_{v}\right\}$ as the set $D$ and $\sim_{R}$ as the equivalence relation $\approx$, this principle gives us a function $f_{\sim_{R}}$ which will serve us as the desired $f_{R}$. Let $D_{R}$ be the range of $f_{R}$; then $f_{R}$ is obviously onto $D_{R}$. For elements $d$ and $e$ of $D_{R}$, define $d>_{R} e$ if and only if there are pairs $\langle a, v\rangle$ and $\langle b, w\rangle$ such that $d=f_{R}(a, v)$, $e=f_{R}(b, w)$, and $\langle a, b\rangle \in R_{v, w}$. It is easy to check that $>_{R}$ is well-defined and a strict linear order. ${ }^{16}$

We have now shown that, once abstraction is granted, the two notions of model are equivalent. ${ }^{17}$ This takes care of the first dimension of comparison mentioned at the beginning of this section; if one accepts the abstraction principle, one kind of structure is as good as the other. The second dimension concerns the choice of modal object language; and here the situation is not symmetric.

As I pointed out above, the proponent of QML has only one option when it comes to the formalization of cross-world predication, namely the inclusion of the requisite sort of degrees in her ontology, and the analysis of

(1a) John might have been taller than Mary is

as

(1R) Mary's height is such that, under certain circumstances, John's height would have been greater than it,

which can then be rendered in the object language as

(1Rf) [the $h: T m h] \diamond\left[\right.$ the $\left.h^{\prime}: T j h^{\prime}\right] h^{\prime}>h$.

Thus this approach cannot avoid an ontological commitment to abstract objects, viz. the degrees associated with the comparative relation: heights in order to deal with "taller than," intelligence quotients, say, for "smarter than," degrees of pulchritude for "prettier than," etc.

On the SML approach, one has three options: First, and least interestingly, one can follow the same path as the QML theorist and simply add Russellian degrees. Clearly this incurs the same ontological commitments. Second, one can instead extend SML to CSML and handle cross-world predication by invoking primitive

\footnotetext{
16 Given a sufficient amount of set theory, the abstraction principle (Abst) can be proved by taking as $f_{\approx}(x)$ the equivalence class of $x$ with respect to $\approx$.

17 This result contrasts with Kemp's (2000) claim that D-structures arise from standard Kripke structures (i.e. structures without cross-world extensions) by applying the process of abstraction at each possible world separately, and then collecting all the $R$-degrees together into one degree set $D_{R}$. This clearly will not work, as separate abstractions in $v$ and $w$ will yield ordered degree sets $\left(D_{R}^{v},>_{R}^{v}\right)$ and $\left(D_{R}^{w},>{ }_{R}^{w}\right)$ relative to $v$ and $w$, but provide no information as to how elements of $D_{R}^{v}$ relate to elements of $D_{R}^{w}$. In other words, piecemeal abstraction fails to provide for calibration of degrees across worlds.
} 
cross-world relations. This option completely eschews any ontological commitments not already present in the original object language, and instead makes do with a more fine-grained notion of extension. Third, one can combine the first two options. Indeed, if one values having degrees around, this third option seems to be the ideal solution.

For consider the inference from (1a) to (1R) again.

The QML analysis has no choice but to treat this as a trivial inference of the form " $A$, therefore $A$," because it must regard (1R) as a mere paraphrase of (1a). But on the face of it, the inference is not trivial-for one thing, it produces a conclusion asserting the existence of certain abstract objects from a premise that does not mention such objects at all. ${ }^{18}$ The framework that combines CSML with Russellian degrees, by contrast, renders the inference in the form

$$
\begin{aligned}
& \diamond R^{s, i} j m \\
& \therefore\left[\text { the } h: T^{i} m h\right] \diamond\left[\text { the } h^{\prime}: T^{s} j h^{\prime}\right] h^{\prime}>h,
\end{aligned}
$$

and thus correctly indicates in the logical forms of the object language its non-trivial nature. Given that QML does not appear to permit a formalization of cross-world predication with this feature, we are therefore inclined to think that the mood-based analysis is the solution of choice.

As a final point in favor of CSML, let us ask which English predicates $R$ are amenable to cross-world predication. As observed by Kemp (2000), it seems to be a necessary condition that $R$ express either a comparative relation or an equivalence relation. But that is clearly not sufficient. Consider, for instance, the following pair of synonymous predicates:

" $x$ is heavier than $y$ is" vs. " $x$ outweighs $y "$

While the former can easily be used for cross-world predication (witness "I might have been heavier than I am"), the latter cannot. Try as you might, the best one can come up with is something like "I might have outweighed myself," which is an intra-world comparison, and indeed a false one.

The reason for this asymmetry is clearly that " $x$ is heavier than $y$ is" possesses two individually inflectible copulas, so that by subjunctivizing one, but not the other, we achieve predication across worlds. On the other hand, " $x$ outweighs $y$ " can only accommodate one grammatical mood, which restricts it to intra-world predication. This logically relevant feature of English and a range of other languages (cf. our examples from German and French) is made explicit in the notation of CSML; however, in the analysis based on traditional QML, it is completely obscured.

Acknowledgments The research reported here was partially supported by a visiting professorship at the Archives Henri Poincaré (UMR 7117 CNRS/Nancy-Université), Université Nancy 2, France. Thanks to Gerhard Heinzmann and Roger Pouivet for the kind reception at the Archives. This essay has grown out of a number of talks (at the Third Irvine-Florence Colloquium in Florence, Italy; a workshop on subjunctive markers at the Université Lille 3, France; the workshop "Independences in Modal Logic" at

\footnotetext{
18 Indeed, as our discussion of D- and CW-structures indicates, the inference rests on an application of the principle (Abst).
} 
the Université Nancy 2, France; and the Universities of Utah, St. Andrews, Oxford, Tübingen, and Heidelberg, as well as CUNY - Graduate Center), and I am grateful to the respective audiences for critical feedback that has improved the paper. Special thanks for helpful comments and criticisms to Aldo Antonelli, John Burgess, Mel Fitting, Volker Halbach, Lloyd Humberstone, Gary Kemp, Cathy Legg, Richard Mendelsohn, Manuel Rebuschi, Helge Rückert, François Schmitz, Peter Schroeder-Heister, Stewart Shapiro, Brian Skyrms, Jordan Stein, Tim Williamson, and Crispin Wright.

Open Access This article is distributed under the terms of the Creative Commons Attribution Noncommercial License which permits any noncommercial use, distribution, and reproduction in any medium, provided the original author(s) and source are credited.

\section{References}

Crossley, J. N. \& Humberstone, L. (1977). The logic of 'Actually'. Reports on Mathematical Logic, 8, $11-29$.

Forbes, G. (1985). The metaphysics of modality. Oxford: Clarendon Press.

Forbes, G. (1989). Languages of possibility. Oxford: Basil Blackwell.

Forbes, G. (1994). Comparatives in counterpart theory: another approach. Analysis, 54(1), 37-42.

Hazen, A. (1990). Actuality and quantification. Notre Dame Journal of Formal Logic, 31, 498-508.

Humberstone, L. (1982). Scope and subjunctivity. Philosophia, 12, 99-126.

Humberstone, L. (2004). Two-dimensional adventures. Philosophical Studies, 118, 17-65.

Kemp, G. (2000). The interpretation of crossworld predication. Philosophical Studies, 98(3), 305-320.

Kripke, S. A. (2005). Russell's notion of scope. Mind, 114, 1005-1037.

Melia, J. (2003). Modality. Montreal and Kingston/Ithaca: McGill-Queen's University Press.

Russell, B. (1905). On denoting. Mind, 14, 479-493.

Wehmeier, K. F. (2003). World travelling and mood swings. In B. Löwe, T. Räsch, W. Malzkorn (Eds.), Foundations of the formal sciences II (pp. 257-260). Dordrecht: Kluwer.

Wehmeier, K. F. (2004). In the mood. Journal of Philosophical Logic, 33(6), 607-630.

Wehmeier, Kai F. (2005). Modality, mood, and descriptions. In R. Kahle (Ed.). Intensionality: An interdisciplinary discussion (pp. 187-216). Wellesley, MA: AK Peters.

Williamson, T. (2006). Indicative versus subjunctive conditionals, congruential versus non-hyperintensional contexts. Philosophical Issues, 16(1), 310-333.

Williamson, T. (2009). Conditionals and actuality. Erkenntnis, 70(2), 135-150. 